\title{
Convergence of a Logistic Type Ultradiscrete Model
}

\author{
Masaki Sekiguchi, ${ }^{1}$ Emiko Ishiwata, ${ }^{2}$ and Yukihiko Nakata $^{3}$ \\ ${ }^{1}$ Tokyo Metropolitan Ogikubo High School, 5-7-20 Ogikubo, Suginami-ku, Tokyo 167-0051, Japan \\ ${ }^{2}$ Department of Applied Mathematics, Tokyo University of Science, 1-3 Kagurazaka, Shinjuku-ku, Tokyo 162-8601, Japan \\ ${ }^{3}$ Department of Mathematics, Shimane University, 1600 Nishikawatsu-cho, Matsue-shi, Shimane 690-8504, Japan
}

Correspondence should be addressed to Masaki Sekiguchi; emafarms@gmail.com

Received 5 May 2017; Accepted 16 July 2017; Published 27 August 2017

Academic Editor: Francisco R. Villatoro

Copyright ( 2017 Masaki Sekiguchi et al. This is an open access article distributed under the Creative Commons Attribution License, which permits unrestricted use, distribution, and reproduction in any medium, provided the original work is properly cited.

We derive a piecewise linear difference equation from logistic equations with time delay by ultradiscretization. The logistic equation that we consider in this paper has been shown to be globally stable in the continuous and discrete time formulations. Here, we study if ultradiscretization preserves the global stability property, analyzing the asymptotic behaviour of the obtained piecewise linear difference equation. It is shown that our piecewise linear difference equation has a threshold property concerning global attractivity of equilibria, similar to the stable logistic equations with time delay.

\section{Introduction}

Ultradiscretization is proposed as a procedure to obtain a discrete system, where unknown variables also take discretized values [1]. The discrete systems are a class of piecewisedefined difference equations [2, 3]. Specifically, ultradiscretization converts addition, multiplication, and division for two numbers in a discrete system into max operator, addition, and subtraction for other two numbers in the ultradiscrete model. Ultradiscrete models are related to the continuous and discrete models via formal solutions and conserved quantities $[1,4]$. See also [5-7] for the application of ultradiscretization to the traffic flow.

In this paper, we consider the following difference equation:

$$
x_{n+1}=\max \left(x_{n}, b+x_{n-\omega}\right)-\max \left(c, b+x_{n-\omega}\right),
$$

where $b$ is a real number and $c$ is a real positive number, with the following initial condition:

$$
x_{-j} \in \mathbb{R} \text { for } j \in\{0,1, \ldots, \omega\} .
$$

We derive the difference equation (1) from a nonlinear difference equation studied in $[8,9]$. The difference equation is an extension of a discrete logistic map and can be seen as a discrete analogue of a disease transmission dynamics model studied in [10]. In Section 2, we briefly introduce the disease transmission dynamics model formulated as a scalar delay differential equation. Subsequently we derive the difference equation (1) from the differential equation via discretization and ultradiscretization. It is known that the applied discretization gives stable numerical solutions [11]. Nonstandard finite difference schemes are used, from a continuous dynamical system, to derive a dynamically consistent discrete system, which preserves qualitative and quantitative properties of the solution of the original continuous differential equation such as positivity, stability of equilibria, and conservation laws; see $[12,13]$ and references therein. The ultradiscrete model (1) is related to two delay equations: delay difference equation studied in $[8,9]$ and delay differential equation studied in [10].

Those delay equations are an extension of a discrete logistic map and the logistic equation, respectively. For the nondelay case, three equations are related to each other, sharing the qualitative property that every solution converges to an equilibrium [14]. It is known that the solution of the discrete model exactly follows the continuous solution of the logistic equation [15]. In the two delay equations, the corresponding equilibria are globally asymptotically stable; thus the discretization preserves the global stability property 
as well as in the nondelay case. In this paper, we study if the difference equation (1) derived from the stable difference and differential equations has a similar property. The convergence property of the difference equation (1) is analyzed in detail.

The paper is organized as follows. In Section 2, we summarize stability of differential and difference logistic equations in [14] and derive our ultradiscrete model from the difference equation. Our objective for this section is clarifying qualitative correspondence between the differential and difference equations. In Section 3, we discuss the convergence property of (1). We prove that the model exhibits the threshold behaviour, similar to the differential equation studied in $[10]$ and the difference equation studied in $[8,9]$. We find here that a subsequence of the solution has a monotone property and this monotonicity is used for the proof. We then summarize our results in Section 4.

\section{Differential and Difference Logistic Equations}

In this section, we summarize the previous studies related to (1).

We start with a logistic equation:

$$
\frac{d y(t)}{d t}=y(t)(\lambda-\delta-\lambda y(t)),
$$

where $\lambda$ and $\delta$ are real positive constants. The reason for the parameterization becomes clear, when we introduce time delay. It is well known that, for the positive initial conditions, the trivial equilibrium, $y=0$, is globally asymptotically stable if $\lambda-\delta \leq 0$, while the positive equilibrium, $y=(\lambda-\delta) / \lambda$, is globally asymptotically stable if $\lambda-\delta>0$.

By an applied discretization [16], the following discrete analogue can be derived from the logistic equation (3):

$$
y_{n+1}=\frac{y_{n}+\beta y_{n}}{1+\gamma+\beta y_{n}} .
$$

Let $h$ be a sufficiently small step size. Then the parameters $\beta$ and $\gamma$ are related to $\lambda$ and $\delta$ via $\beta=h \lambda$ and $\gamma=h \delta$. The difference equation (4) captures the continuous solution of the differential equation (3); that is, the solution shows the logistic curve [15]. See also [14].

The author in [14] obtains the following piecewise linear difference equation from (4) by ultradiscretization:

$$
x_{n+1}=b-c-\max \left(-x_{n}, b-c\right),
$$

where $b$ and $c$ are constants that satisfied $b-c \geq 0$. Ultradiscretization is proposed as a procedure to obtain the discrete system, where unknown variables also take discretized values [1]. In [14], it is shown that the three models (3), (4), and (5) share the qualitative property that every solution converges to an equilibrium.

Following [14, 17-19], let us derive (5) from (4). For $\varepsilon>0$, we introduce a variable $x$ via

$$
y_{n}=e^{x_{n} / \varepsilon}
$$

and parameters $b$ and $c>0$ through

$$
\begin{aligned}
\beta & =e^{b / \varepsilon}, \\
1+\gamma & =e^{c / \varepsilon} .
\end{aligned}
$$

Then we have

$$
e^{x_{n+1} / \varepsilon}=\frac{e^{x_{n} / \varepsilon}\left(1+e^{b / \varepsilon}\right)}{e^{c / \varepsilon}+e^{\left(b+x_{n}\right) / \varepsilon}}
$$

thus

$$
x_{n+1}=x_{n}+\varepsilon \log \left(1+e^{b / \varepsilon}\right)-\varepsilon \log \left(e^{c / \varepsilon}+e^{\left(b+x_{n}\right) / \varepsilon}\right) .
$$

Letting $\varepsilon \rightarrow+0$ and assuming $b>0$, we get (5) by the following manipulations:

$$
\begin{aligned}
x_{n+1} & =x_{n}+\max (0, b)-\max \left(c, b+x_{n}\right) \\
& =b-\max \left(c-x_{n}, b\right) \\
& =b-c-\max \left(-x_{n}, b-c\right) .
\end{aligned}
$$

The key relation used here is the following limit:

$$
\lim _{\varepsilon \rightarrow+0} \varepsilon \log \left(e^{A / \varepsilon}+e^{B / \varepsilon}\right)=\max (A, B)
$$

for $A, B>0$.

An epidemic model considered in [10] is an extension of the logistic equation (3). The model is formulated as the following delay differential equation:

$$
\frac{d y(t)}{d t}=\lambda y(t-\tau)(1-y(t))-\delta y(t),
$$

where $\tau$ is a real positive constant. The global stability condition for (12) is the same as the condition for the nondelay case (3): for the positive initial conditions, the trivial equilibrium, $y=0$, is globally asymptotically stable if $\lambda-\delta \leq 0$, while the positive equilibrium, $y=(\lambda-\delta) / \lambda$, is globally asymptotically stable if $\lambda-\delta>0$. Different logistic equations with time delay have the instability property; see [20-22].

In order to ensure positivity of the solution in discrete analogues of the differential equation (11), we use Mickens nonstandard finite difference scheme [11] to discretize (12) as follows:

$$
\begin{aligned}
\frac{y(t+h)-y(t)}{h}= & \lambda y(t-\tau)(1-y(t+h)) \\
& -\delta y(t+h),
\end{aligned}
$$

where $h>0$ is a step size. Equation (13) can be written by the following explicit form:

$$
y(t+h)=\frac{y(t)+h \lambda y(t-\tau)}{1+h \delta+h \lambda y(t-\tau)}
$$

thus (13) is equivalently written as the following difference equation with $h \delta=\gamma, h \lambda=\beta, y(t)=y_{n}$, and $y(t+h)=y_{n+1}$ :

$$
y_{n+1}=\frac{y_{n}+\beta y_{n-\omega}}{1+\gamma+\beta y_{n-\omega}},
$$


where $\beta$ and $\gamma$ are positive constants and $\omega$ is a nonnegative integer. It is obvious that the delay equation (15) is reduced to (4) when $\omega=0$. Equation (15) is a special case of the model considered in $[8,9]$. For some specific and general cases, the authors in [23-26] show global asymptotic stabilities of the zero and positive equilibria. The zero equilibrium of (15) is globally asymptotically stable when $\beta \leq \gamma$. The unique equilibrium of (15) is globally asymptotically stable when $\beta>\gamma$. From those results, the difference equation (15) can be seen as a discrete analogue that preserves the global stability property of (12).

Let us now derive the difference equation (1) from (15). For $\varepsilon>0$, we introduce the variable $x$ and the parameters $b$ and $c$ in the same way as the derivation of (5); then we get

$$
\begin{aligned}
x_{n+1}= & \varepsilon \log \left(e^{x_{n} / \varepsilon}+e^{\left(b+x_{n-\omega}\right) / \varepsilon}\right) \\
& -\varepsilon \log \left(e^{c / \varepsilon}+e^{\left(b+x_{n-\omega}\right) / \varepsilon}\right) .
\end{aligned}
$$

Letting $\varepsilon \rightarrow+0$ and using the key relation (11), we get (1). Finally, we note that (5) is a special case of (1). In fact, let $\omega=0$ and $b>0$ in (5). Then

$$
\begin{aligned}
x_{n+1} & =\max \left(x_{n}, b+x_{n}\right)-\max \left(c, b+x_{n}\right) \\
& =x_{n}+\max (0, b)-x_{n}-\max \left(c-x_{n}, b\right) \\
& =b-c-\max \left(-x_{n}, b-c\right) .
\end{aligned}
$$

In the following section, we study the convergence of the solution of (1).

\section{Global Properties of the Solution}

In this section, we elucidate that the three models (12), (15), and (1) have the same qualitative properties. To do that, we study the asymptotic behaviour of the solutions of (1).

Lemma 1. For any solution, there exists $\bar{n} \in \mathbb{N}_{+}$such that $x_{n} \leq$ 0 for $n \geq \bar{n}$.

Proof. Let us assume that $x_{n} \geq c$ for some $n \geq 0$. Then

$$
\begin{aligned}
\max \left(x_{n}, b+x_{n-\omega}\right)= & x_{n}-c \\
& +\max \left(c,-x_{n}+c+b+x_{n-\omega}\right) \\
\leq & x_{n}-c+\max \left(c, b+x_{n-\omega}\right) .
\end{aligned}
$$

Using this estimation in (1), we get

$$
\begin{aligned}
x_{n+1} & \leq x_{n}-c+\max \left(c, b+x_{n-\omega}\right)-\max \left(c, b+x_{n-\omega}\right) \\
& =x_{n}-c .
\end{aligned}
$$

This implies that $x_{n}$ is decreasing with respect to $n$ as long as $\mathrm{x}_{n} \geq c$. Therefore, there exists $k$ such that $x_{k-1} \geq c$ and $x_{k}<c$. Then from (1) with $n=k$, it follows that

$$
x_{k+1} \leq \max \left(c, b+x_{k-\omega}\right)-\max \left(c, b+x_{k-\omega}\right)=0 .
$$

Inductively we get that $x_{m} \leq 0$ for all $m \geq k+1=\bar{n}$.
From Lemma 1, without loss of generality, we can set the initial condition as

$$
x_{-j} \leq 0, \quad j \in\{0,1, \ldots, \omega\} .
$$

Note that Lemma 1 implies that

$$
\{x \in \mathbb{R} \mid x \leq 0\}
$$

is an invariant set.

To discuss global attractivity of equilibria of the scalar difference equation (1), it seems to be convenient to consider an equivalent two-dimensional system. From (22) and $c>0$, one has

$$
b+x_{n-\omega} \geq x_{n}-c+b+x_{n-\omega},
$$

and then we can write

$$
\begin{aligned}
& \max \left(x_{n}, b+x_{n-\omega}\right) \\
& \quad=\max \left(x_{n}, x_{n}-c+b+x_{n-\omega}, b+x_{n-\omega}\right) .
\end{aligned}
$$

Now we define $w_{n+1}=-\max \left(0,-c+b+x_{n-\omega}\right)$; then from (1) and (24) one has

$$
\begin{aligned}
x_{n+1}= & \max \left(x_{n}, x_{n}-c+b+x_{n-\omega}, b+x_{n-\omega}\right)+w_{n+1} \\
& -c \\
= & \max \left(x_{n}-w_{n+1}, b+x_{n-\omega}\right)+w_{n+1}-c \\
= & \max \left(x_{n}, b+x_{n-\omega}+w_{n+1}\right)-c,
\end{aligned}
$$

where we use $w_{n+1} \leq 0$ in (22). Therefore we can get the following system:

$$
\begin{aligned}
& w_{n+1}=-\max \left(0,-c+b+x_{n-\omega}\right), \\
& x_{n+1}=\max \left(x_{n}, b+x_{n-\omega}+w_{n+1}\right)-c .
\end{aligned}
$$

The initial condition is given as (22).

To discuss global attractivity of equilibria, we now consider (26a) and (26b) in the set given as in (22).

Theorem 2. Let one assume that $b<c$ holds. Then

$$
\begin{aligned}
w_{n} & =0 \quad \text { for } n \geq 1, \\
\lim _{n \rightarrow \infty} x_{n} & =-\infty .
\end{aligned}
$$

Proof. Since for any $n \geq 0$ one has that $-c+b+x_{n-\omega} \leq b-c<0$ from Lemma 1, it follows that

$$
w_{n+1}=-\max \left(0,-c+b+x_{n-\omega}\right)=0 .
$$

Therefore it follows that $w_{n+1}=0$ for any $n \geq 0$ from (26a). From (26b), we get

$$
x_{n+1}=\max \left(x_{n}-c, x_{n-\omega}+b-c\right) .
$$

Let

$$
\widehat{x}_{m}:=\max _{0 \leq j \leq \omega} x_{m(\omega+1)-j} \quad \text { for } m \in \mathbb{N}_{+} .
$$


Note that $x_{m(\omega+1)-j} \leq \widehat{x}_{m}$ for $j \in\{0,1, \ldots, \omega\}$. We show that

$$
\widehat{x}_{m+1} \leq \widehat{x}_{m}+\max (-c, b-c) .
$$

From (29), we have

$$
\begin{aligned}
x_{(m+1)(\omega+1)-\omega} & =x_{m(\omega+1)+1} \\
& =\max \left(x_{m(\omega+1)}-c, x_{m(\omega+1)-\omega}+b-c\right) \\
& \leq \max \left(\widehat{x}_{m}-c, \widehat{x}_{m}+b-c\right) \\
& =\widehat{x}_{m}+\max (-c, b-c) .
\end{aligned}
$$

For some $j \in\{1,2, \ldots, \omega\}$, suppose that

$$
x_{(m+1)(\omega+1)-j} \leq \widehat{x}_{m}+\max (-c, b-c) .
$$

Then using (29) and (33), we obtain

$$
\begin{aligned}
& x_{(m+1)(\omega+1)-j+1} \\
& \quad=\max \left(x_{(m+1)(\omega+1)-j}-c, x_{m(\omega+1)-j+1}+b-c\right) \\
& \quad \leq \widehat{x}_{m}+\max (-c, b-c) .
\end{aligned}
$$

By mathematical induction, it holds that $x_{(m+1)(\omega+1)-j} \leq \hat{x}_{m}+$ $\max (-c, b-c)$ for any $j \in\{0,1, \ldots, \omega\}$. Therefore we get

$$
\widehat{x}_{m+1} \leq \widehat{x}_{m}+\max (-c, b-c) .
$$

Now it is obvious that $\lim _{m \rightarrow \infty} \widehat{x}_{m}=-\infty$ and hence $\lim _{n \rightarrow \infty} x_{n}=-\infty$. We thus obtain the conclusion.

If $b>c, w_{n}$ and $x_{n}$ converge to a unique equilibrium. First we show that system (26a) and (26b) has a nontrivial equilibrium.

Proposition 3. Let one assume that $b>c$ holds. Then system (26a) and (26b) has an equilibrium $(-b+c, 0)$.

Proof. Let $b>c>0$ holds. We show that system (26a) and (26b) has the constant solution $(-b+c, 0)$. From direct computations, one can see

$$
\begin{gathered}
-\max (0,-c+b)=-b+c, \\
\max (0, c)-c=0 .
\end{gathered}
$$

Proposition 4. Let one assume that $b>c$ holds. It follows that

$$
\begin{aligned}
& x_{n+1} \\
& \quad= \begin{cases}0 & \text { if } x_{n-\omega} \geq-b+c \\
\max \left(x_{n}-c, x_{n-\omega}+b-c\right) & \text { if } x_{n-\omega}<-b+c .\end{cases}
\end{aligned}
$$

Proof. Assume that $x_{n-\omega} \geq-b+c$. Then it is straightforward to get $w_{n+1}=c-b-x_{n-\omega}$ from (26a). Since we have $x_{n} \leq 0$ (see Lemma 1), we get

$$
x_{n+1}=\max \left(x_{n}, c\right)-c=0 .
$$

On the other hand, assume that $x_{n-\omega}<-b+c$. Then $w_{n+1}=$ 0 follows from (26a). Thus we immediately obtain the conclusion from (26b) with $w_{n+1}=0$.
We now show that every solution converges to the nontrivial equilibrium.

Theorem 5. Let us assume that $b>c$. Then

$$
\begin{aligned}
& \lim _{n \rightarrow \infty} w_{n}=-b+c, \\
& \lim _{n \rightarrow \infty} x_{n}=0 .
\end{aligned}
$$

Proof. Let

$$
\mathbf{x}_{\ell}:=\left(x_{\ell(\omega+1)}, x_{\ell(\omega+1)-1}, \ldots, x_{\ell(\omega+1)-\omega}\right)
$$

for $\ell \in \mathbb{N}_{+}$. From Proposition 4, one can see that

$$
x_{\ell(\omega+1)-k} \geq b-c+x_{(\ell-1)(\omega+1)-k}
$$

for $k \in\{0,1,2, \ldots, \omega\}$ if $x_{(\ell-1)(\omega+1)-k}<-b+c$. Therefore,

$$
\lim _{\ell \rightarrow \infty} x_{\ell(\omega+1)-k}=0, \quad k \in\{0,1,2, \ldots, \omega\}
$$

that is, each component of $\mathbf{x}_{\ell}$ converges to the equilibrium as $\ell \rightarrow \infty$. Then, there exists a sufficiently large integer $m$ such that $x_{n}=\cdots=x_{n-\omega}=0$ for $n \geq m$. For $n \geq m$, we obtain

$$
w_{n+1}=-\max (0,-c+b)=-b+c \text {. }
$$

Theorems 2 and 5 show that $b<c$ and $b>c$ are, respectively, the criteria of the global divergence to $-\infty$ and the convergence to the unique equilibrium. Equation (1) also has the threshold dynamics as in (12) and (15).

For $Y=\left(y_{1}, y_{2}, \ldots, y_{\omega+1}\right) \in \mathbb{R}^{\omega+1}$, we define $\|Y\|=$ $\sqrt{\sum_{j=1}^{\omega+1} y_{j}^{2}}$. Let

$$
X_{n}:=\left(x_{n}, x_{n-1}, \ldots, x_{n-\omega}\right) .
$$

Here we show that the equilibrium is stable. Assume that $x_{n-\omega}>0$. If $\left\|X_{n}\right\|<c$, then from (1) we obtain $x_{n+1}=0$. Thus

$$
\left\|X_{n}\right\| \geq\left\|X_{n+1}\right\|
$$

follows. On the other hand, assume that $x_{n-\omega} \leq 0$. If $\left\|X_{n}\right\|<$ $b-c$, then, from Proposition 4 , we obtain $x_{n+1}=0$. Thus (45) follows. Consequently, if $\left\|X_{n}\right\|<\min (c, b-c)$, then we obtain (45). Thus the equilibrium is stable in $\mathbb{R}^{\omega+1}$.

In Figure 1, we plot $x_{n}$ with respect to $n$. For $\omega=6$, the initial condition is chosen as

$$
\begin{aligned}
& \left(x_{-6}, x_{-5}, x_{-4}, x_{-3}, x_{-2}, x_{-1}, x_{0}\right) \\
& \quad=(-10,-11,-20,-13,-20,-15,-16) .
\end{aligned}
$$

We set the parameters as $b=1$ and $c=3$ in Figure 1(a), while $b=5$ and $c=2$ in Figure 1(b). As in Theorems 2 and 5, one can see that $x$ tends to $-\infty$ as $n \rightarrow \infty$ in Figure 1(a) and that $x$ tends to 0 as $n \rightarrow \infty$ in Figure 1(b). 


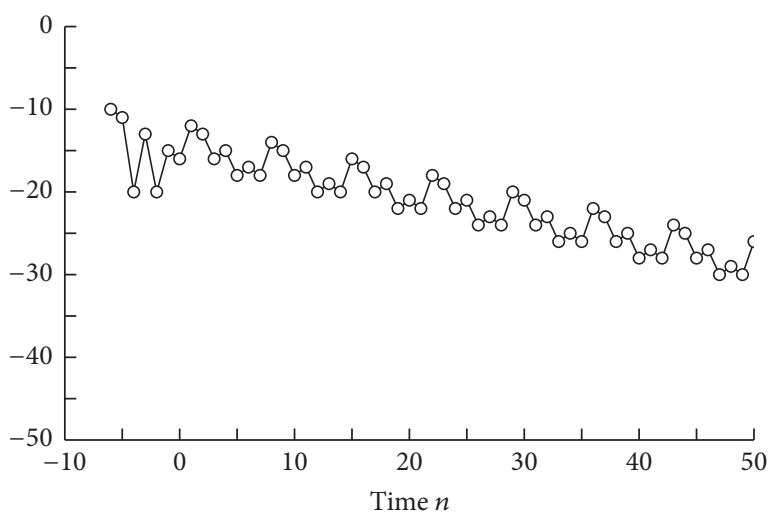

$\multimap-$ Number of $x_{n}$

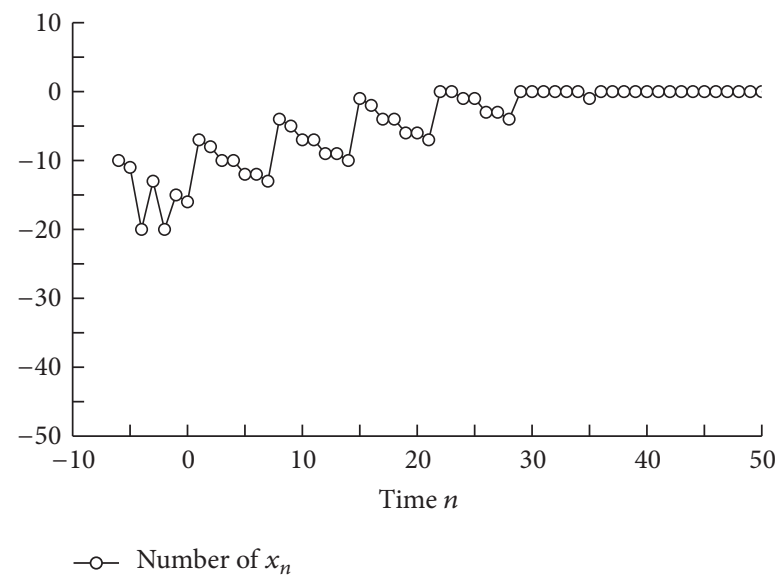

(b) $b>c$ $\multimap-$ Number of $x_{n}$

(a) $b<c$

FIGURE 1: Numerical illustration of a solution behaviour $(\omega=6)$.

\section{Conclusion}

In this paper, we consider an ultradiscrete model with time delay. In Theorems 2 and 5, we show that the ultradiscrete model also has the threshold property concerning global attractivity of equilibria, similar to the difference equation [8] and differential equation [10]. For the proof of global attractivity of the nontrivial equilibrium in Theorem 5, we reduce system (26a) and (26b) to the scalar difference equation in Proposition 4 and then use a certain monotone property of the solution.

In a different study, the scalar difference equation system (26a) and (26b) also appears, where we derive an ultradiscrete model from an SIR type epidemic model. In the SIR type epidemic model, no reinfection is assumed after the recovery [27], differently from the assumption of the SIS type epidemic model. Although those model structures are different, we encounter the same difference equation system (26a) and (26b) in the ultradiscrete level. The implication shall be explored in the future study.

In this paper, we study qualitative properties of the ultradiscrete model (1). In [19], it is shown that simple ultradiscrete models can capture disease transmission dynamics. Cellular automata have been used to model complex phenomena including disease transmission dynamics. Since cellular automata are computational models, in general, it is not straightforward to perform a mathematical analysis, in order to provide theoretical basis for the simulation studies. Our analytical study for the ultradiscrete model could be complement for numerical simulation studies for some cellular automaton models.

\section{Conflicts of Interest}

The authors declare that they have no conflicts of interest.

\section{Acknowledgments}

The second author was supported by JSPS Grant-in-Aid for Scientific Research (C) JP26400212. The third author was supported by JSPS Grant-in-Aid for Young Scientists (B) $16 \mathrm{~K} 20976$.

\section{References}

[1] T. Tokihiro, D. Takahashi, J. Matsukidaira, and J. Satsuma, "From soliton equations to integrable cellular automata through a limiting procedure," Physical Review Letters, vol. 76, no. 18, pp. 3247-3250, 1996.

[2] C. M. Kent, "Piecewise-defined difference equations: open problem," in Bridging Mathematics, Statistics, Engineering and Technology, B. Toni, K. Williamson, N. Ghariban, D. Haile, and Z. Xie, Eds., vol. 24 of Springer Proceedings in Mathematics \& Statistics, Springer, New York, NY, USA, 2012.

[3] W. Tikjha, E. Lapierre, and T. Sitthiwirattham, "The stable equilibrium of a system of piecewise linear difference equations," Advances in Difference Equations, 2017.

[4] A. Nagai, T. Tokihiro, and J. Satsuma, "Conserved quantities of box and ball system," Glasgow Mathematical Journal, vol. 43A, pp. 91-97, 2001.

[5] K. Nishinari and D. Takahashi, "Analytical properties of ultradiscrete Burgers equation and rule-184 cellular automaton," Journal of Physics A, vol. 31, no. 24, pp. 5439-5450, 1998.

[6] K. Nishinari and D. Takahashi, "A new deterministic CA model for traffic flow with multiple states," Journal of Physics A, vol. 32, no. 1, pp. 93-104, 1999.

[7] K. Matsuya and M. Kanai, Exact solution of a delay difference equation modeling traffic flow and their ultra-discrete limit, https://arxiv.org/abs/1509.07861.

[8] E. Chatterjee, "On the global character of the solutions of $X_{n+1}=$ $\left(\alpha+\beta X_{n}+\gamma X_{n-k}\right) /\left(A+X_{n-k}\right)$," International Journal of Applied Mathematics, vol. 26, no. 1, pp. 9-17, 2013.

[9] C. Zhang and H.-X. Li, "Dynamics of a rational difference equation of higher order," Applied Mathematics E-Notes, vol. 9, pp. 80-88, 2009.

[10] K. L. Cooke, "Stability analysis for a vector disease model," The Rocky Mountain Journal of Mathematics, vol. 9, no. 1, pp. 31-42, 1979.

[11] R. E. Mickens, "Discretizations of nonlinear differential equations using explicit nonstandard methods," Journal of Computational and Applied Mathematics, vol. 110, no. 1, pp. 181-185, 1999. 
[12] R. Anguelov, J. M. Lubuma, and M. Shillor, "Dynamically consistent nonstandard finite difference schemes for continuous dynamical systems," Discrete and Continuous Dynamical Systems. Series A, pp. 34-43, 2009.

[13] L. W. Roeger, "Dynamically consistent discrete-time SI and SIS epidemic models," Discrete and Continuous Dynamical Systems. Series A, pp. 653-662, 2013.

[14] R. Willox, "Modelling natural phenomena with discrete and ultradiscrete systems," in Proceedings of the RIAM Symposium Held at Chikushi Campus, Kyushu Universiy 22AO-S8, pp. 1322.

[15] M. Morisita, "The fitting of the logistic equation to the rate of increase of population density," Researches on Population Ecology, vol. 7, no. 1, pp. 52-55, 1965.

[16] S. Elaydi, An Introduction t o Difference Equations, Springer, New York, NY, USA, 2005.

[17] R. Willox, B. Grammaticos, A. S. Carstea, and A. Ramani, "Epidemic dynamics: discrete-time and cellular automaton models," Physica A, vol. 328, no. 1-2, pp. 13-22, 2003.

[18] J. Satsuma, R. Willox, A. Ramani, B. Grammaticos, and A. S. Carstea, "Extending the SIR epidemic model," Physica A, vol. 336, no. 3-4, pp. 369-375, 2004.

[19] A. Ramani, A. S. Carstea, R. Willox, and B. Grammaticos, "Oscillating epidemics: a discrete-time model," Physica A, vol. 333, no. 1-4, pp. 278-292, 2004.

[20] K. Cooke, P. van den Driessche, and X. Zou, "Interaction of maturation delay and nonlinear birth in population and epidemic models," Journal of Mathematical Biology, vol. 39, no. 4, pp. 332-352, 1999.

[21] G. Huang, A. Liu, and U. Forys, "Global stability analysis of some nonlinear delay differential equations in population dynamics," Journal of Nonlinear Science, vol. 26, no. 1, pp. 2741, 2016.

[22] S. Ruan, "Delay differential equations in single species dynamics," Delay Differential Equations and Applications, vol. 205, pp. 477-517, 2006.

[23] M. R. Kulenović, G. Ladas, and N. R. Prokup, "A rational difference equation," Computers \& Mathematics with Applications, vol. 41, no. 5-6, pp. 671-678, 2001.

[24] E. Camouzis and G. Ladas, Dynamics of third-order rational difference equations with open problems and conjectures, vol. 5 of Advances in Discrete Mathematics and Applications, Chapman \& Hall/CRC Press, Boca Raton, FL, USA, 2008.

[25] W.-T. Li and H.-R. Sun, "Dynamics of a rational difference equation," Applied Mathematics and Computation, vol. 163, no. 2, pp. 577-591, 2005.

[26] V. L. Kocić and G. Ladas, "Global attractivity in nonlinear delay difference equations," Proceedings of the American Mathematical Society, vol. 115, no. 4, pp. 1083-1088, 1992.

[27] H. W. Hethcote, "Qualitative analyses of communicable disease models," Mathematical Biosciences, vol. 28, no. 3/4, pp. 335-356, 1976. 


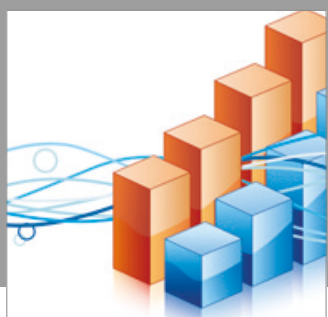

Advances in

Operations Research

vatersals

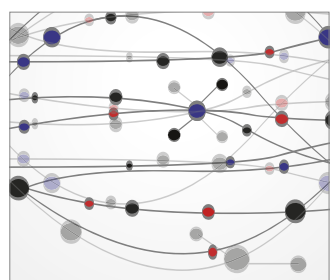

\section{The Scientific} World Journal
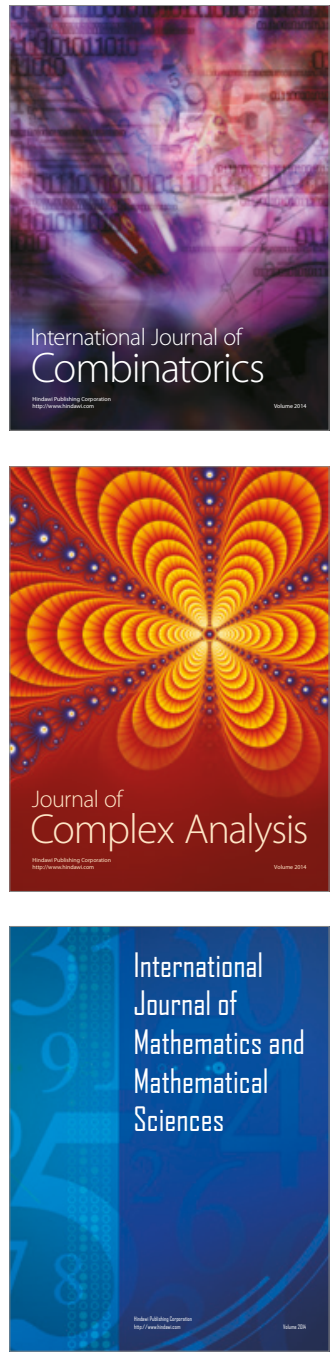
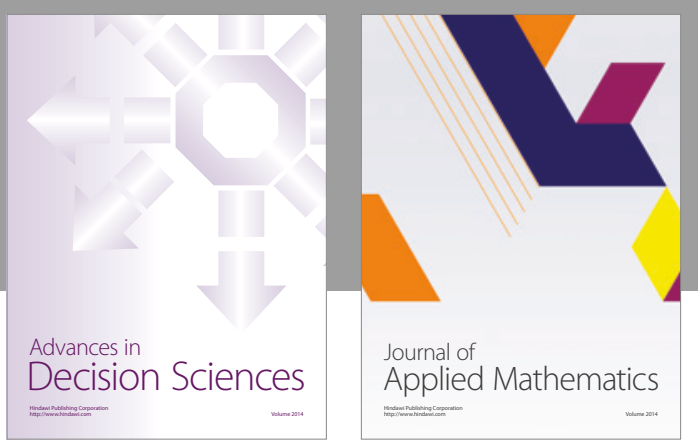

Algebra

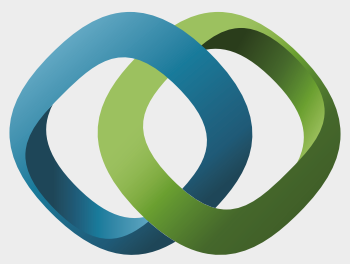

\section{Hindawi}

Submit your manuscripts at

https://www.hindawi.com
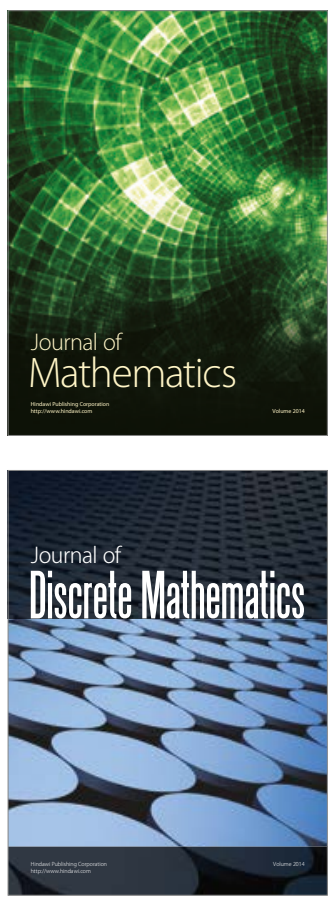

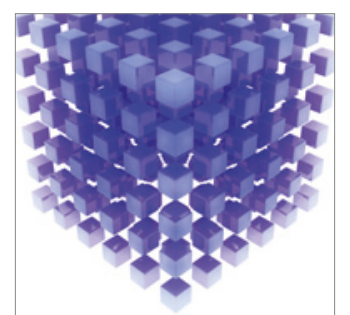

Mathematical Problems in Engineering
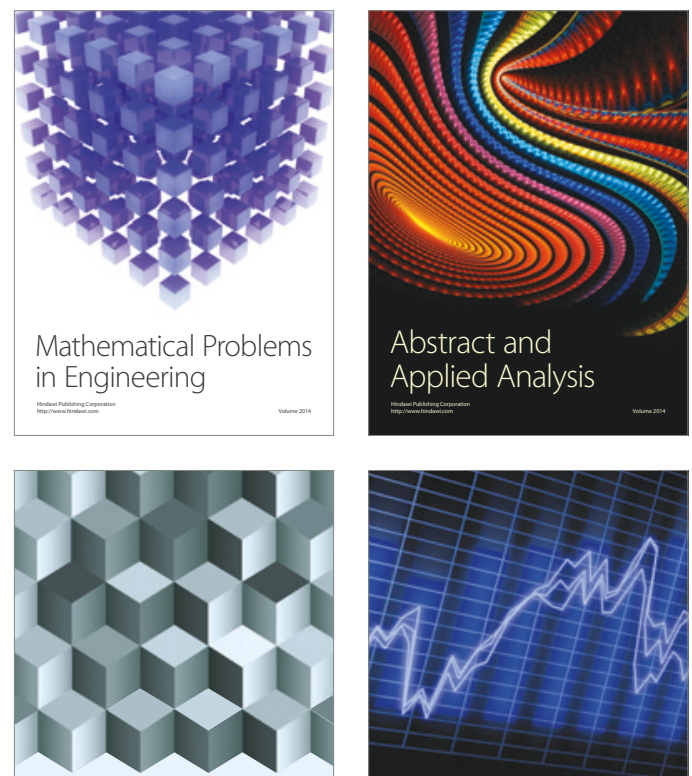

Journal of

Function Spaces

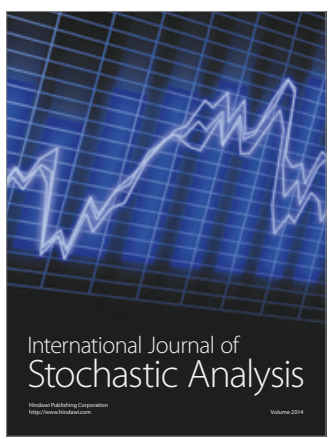

Probability and Statistics
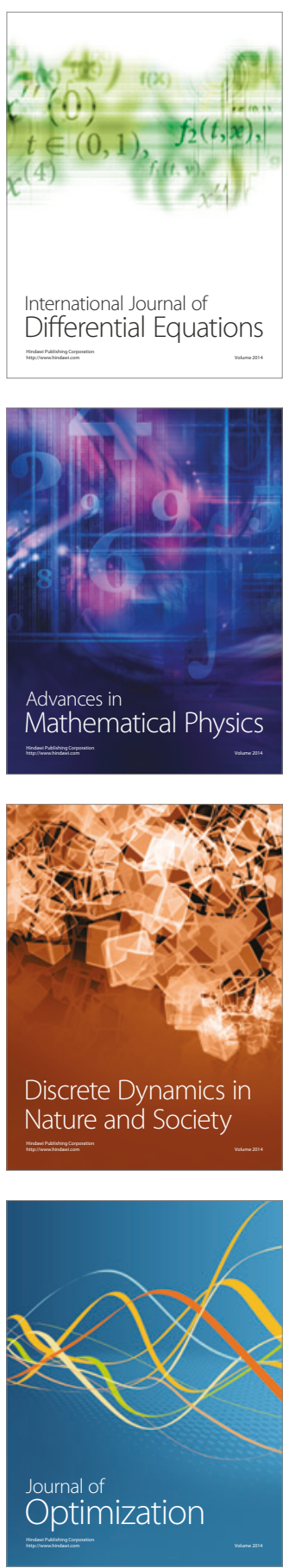